\title{
Assessment of the impact of hereditary factors on biochemical parameters of cardiovascular risk in relation to moderate alcohol consumption
}

\author{
M.J. Kotze ${ }^{\mathrm{a}, *}$, J.L. Marnewick ${ }^{\mathrm{b}}$, M. Kidd ${ }^{\mathrm{c}}$, L.R. Fisher ${ }^{\mathrm{a}}$ and D.P. van Velden ${ }^{\mathrm{a}}$ \\ ${ }^{a}$ Faculty of Medicine and Health Sciences, Department of Pathology, University of Stellenbosch, \\ Tygerberg, South Africa \\ ${ }^{\mathrm{b}}$ Faculty of Health and Wellness Sciences, Oxidative Stress Research Centre, Cape Peninsula University of \\ Technology, Cape Town, South Africa \\ ${ }^{\mathrm{c}}$ Centre for Statistical Consultation, University of Stellenbosch, Tygerberg, South Africa
}

\begin{abstract}
Alcohol intake is associated with variation in many biochemical markers and metabolic processes implicated in cardiovascular disease (CVD) and other non-communicable diseases associated with advanced aging. Some changes are beneficial, while others may be harmful in relation to individual differences in genetic background. The objective of this study was to compare the effects of alternative moderate consumption of red wine and brandy on the lipid profile in the same experimental population of healthy adults and to identify genetic risk factors that may contribute to differences in response. An 8-week randomised crossover intervention study of red wine versus brandy consumption was performed in 37 healthy volunteers (19 males and 18 females) between the ages of 26 and 71 years. The assessments included anthropometric measurements, biochemical determinations and genetic analysis using a multi-gene CVD test. The level of total glutathione as an indicator of antioxidant capacity was significantly decreased only after the brandy intervention when compared to the baseline $(p=0.002)$. Both the brandy and wine intervention resulted in a significant increase in HDL-cholesterol $(p<0.001)$. Significant differential effects were shown in relation to genetic variation in the APOE $(p=0.027)$ and MTHFR $(p=0.048)$ genes, known to be associated with hyper-responsiveness to alcohol intake. Novel findings included a statistically significant increase of both total cholesterol $(p=0.016)$ and triglyceride $(p=0.022)$ levels with alcohol intake only in individuals who tested positive for the low-penetrance $\mathrm{H} 63 \mathrm{D}$ and/or $\mathrm{C} 282 \mathrm{Y}$ mutations in the HFE gene. This study confirmed the well-established protective effect of moderate alcohol consumption on the lipid profile, especially red wine. Since the genetic background influences the effect of alcohol on biochemical parameters of CVD risk, safe limits of wine and brandy consumption may in future be based partly on the genetic profile.
\end{abstract}

Keywords: Alcohol, antioxidant, cardiovascular risk, genetics

\section{Introduction}

As indicated by various scientific studies, regular and moderate wine consumption, particularly red wine, reduces cardiovascular mortality and the incidence of

*Corresponding author: M.J. Kotze, Faculty of Medicine and Health Sciences, Department of Pathology, University of Stellenbosch, Tygerberg, South Africa. Tel.: +27 21 9389324; Fax: +0866009434; E-mail: maritha@sun.ac.za. diabetes. However, whether these effects are due to alcohol or to non-alcoholic components of red wine still remains unknown. In 1992 Renaud and de Lorgeril wrote in The Lancet and eternalised the unforgettable phrase, the "French Paradox". In most countries, high intake of saturated fat is positively related to high mortality from coronary heart disease (CHD). However, the situation in France is paradoxical in that there is high intake of saturated fat but low mortality from CHD. 
This paradox may be attributable in part to the protective effects of regular wine consumption [1]. Thus by adding wine to the protection afforded by high dietary intake of fresh vegetables and olive oil, a hypothesis with wider implication emerged: prediction of CHD involving dietary factors extending beyond the established risk factors.

Traditionally, the French enjoy growing their own vegetables in their gardens. Eating fresh vegetables plus the exercise associated with gardening may both contribute to protection against the adverse effects of the traditional high fat diet and may help to explain the French Paradox. It is now generally accepted that the Mediterranean diet confers health benefits and that wine with meals is part of that diet [2]. Although the definitions of the Mediterranean diet differ, there is consensus that the Mediterranean-style diet is cardioprotective [2].

There are three important points regarding the French food pattern to take into consideration. First, the diet is very varied. Diets with low diversity are associated with increased CHD mortality [3]. Secondly, the pattern of drinking is very different. In France, wine is consumed daily and during meals, rarely alone [1]. Thirdly, devotion to food is a major part of the French culture and choice of food is pleasure. Unfortunately, even in France habits change with the times. Meals are becoming shorter and less elaborate. Less exercise is another current trend and as gardening disappears, so does the French Paradox weaken. The most recent prospective population study shows that the French (or, at least, those studied) have similar cardiovascular outcomes to those calculated from traditional Framingham-based risk factor scores that are regarded as valid throughout the rest of the Western world [4].

Previous studies performed in South Africa support the protective effects of red wine on biochemical markers of CHD [5], while the genetic contribution to these parameters may influence the response to dietary factors and alcohol consumption [6, 7]. We have also previously confirmed the known deleterious effect of genetic variation in the apolipoprotein $\mathrm{E}(A P O E)$ gene on cholesterol levels [8] and development of the metabolic syndrome [9] in the South African population. This is the first study to compare the effect of the alternative consumption of brandy and wine consumption on certain health parameters in the context of a newly-developed pathology-supported genetic testing strategy aimed at prevention of cumulative risk and healthy aging $[10,11]$. Since brandy contains much less non-alcoholic components than wine, it is important to determine whether moderate intake of these beverages have similar cardiovascular protective properties. Whether genetic variation contributes to differences in responses to alcohol use also remains an open question [12].

\section{Research design and methods}

\subsection{Ethics approval}

The study protocol (N09/08/225) was approved by the Ethics Review Committee of the University of Stellenbosch. Written informed consent for participation in the study was obtained after informing the study participants that the e4 allele of the APOE gene included in the genetic analysis does not only increase the risk of CHD in the presence of environmental risk factors such as smoking and a high-saturated fat diet, but also the risk of Alzheimer's disease, with a specific gene-alcohol effect documented in various studies. As reviewed by Kotze and van Rensburg [10], potential gene-environment interaction had to be carefully considered before the genetic test results were provided to the participants using our pathology supported genetic testing strategy.

\subsection{Study population}

The study population included 37 healthy volunteers: 19 males between the ages of 26 and 71 years (average age $50.37 \pm 12.40$ ) and 18 females between the ages of 26 and 69 years (average age $48.61 \pm 12.13)$. Patients with monogenic disorders of lipoprotein metabolism, renal disorders, hepatic disease, morbid obesity, diabetes, hypertension requiring medical treatment or a family history of alcoholism were excluded. Daily intake of dietary supplements, vaso-active medications, lipid lowering agents and smoking were defined as exclusion criteria and study participants were also encouraged to continue with their current eating patterns during the study period.

\subsection{Anthropometric measurements}

Blood pressure, body weight, height as well as waist and hip circumferences were measured at baseline and follow-up visits of participants. Body mass index (BMI) and the waist/hip ratio (WHR) were calculated. 
Table 1

Summary of the low-penetrance mutations evaluated as part of the CVD multi-gene test in this study and their metabolic associations

\begin{tabular}{lll}
\hline Biological pathway & Gene & Genetic variation \\
\hline Lipid and Lipoprotein metabolism & APOE & 3937 T>C, allele e4 (rs429358) \\
& & $4075 \mathrm{C}>\mathrm{T}$, allele e2 (rs7412) \\
Homocysteine and Folate metabolism & MTHFR & $677 \mathrm{C}>\mathrm{T}, \mathrm{A} 222 \mathrm{~V}(\mathrm{rs} 1801133)$ \\
Inflammation & & $1298 \mathrm{~A}>\mathrm{C}, \mathrm{E} 429 \mathrm{~A}(\mathrm{rs} 1801131)$ \\
Haemostasis & Factor II & $20210 \mathrm{G}>\mathrm{A}(\mathrm{rs} 1799963)$ \\
& Factor V & $1691 \mathrm{G}>\mathrm{A}, \mathrm{Leiden}(\mathrm{rs6025})$ \\
Iron metabolism & HFE & $845 \mathrm{G}>\mathrm{A}, \mathrm{C} 282 \mathrm{Y}(\mathrm{rs} 1800562)$ \\
Oxidative stress & & $187 \mathrm{C}>\mathrm{G}, \mathrm{H} 63 \mathrm{D}(\mathrm{rs} 1799945)$ \\
\hline
\end{tabular}

\subsection{Randomized controlled crossover intervention of wine and brandy}

After a 2-week washout period, half of the participants consumed $250 / 175 \mathrm{ml}$ red wine per day $\times 28$ days, and the other half consumed $50 / 40 \mathrm{ml}$ per day brandy $\times 28$ days, respectively, for males and females. This intervention was followed immediately by a crossover period of wine and brandy consumption for 28 days. Wine consumption in males was $250 \mathrm{ml} @ 13.5 \%$ alcohol=33.75 g alcohol per day $(13.5 \times 2.5=33.75 \mathrm{~g})$ and in females $175 \mathrm{ml} @ 13.5 \%$ alcohol=23.63 g alcohol per day $(13.5 \times 1.75=23.63 \mathrm{~g}) \quad$ (or $0.35 \mathrm{~g}$ alcohol $/ \mathrm{kg}$ body mass for males and females). Brandy consumption in males was: $50 \mathrm{ml} @ 43 \%$ alcohol=21.5 g alcohol per day $(43 \times 0.5=21.5 \mathrm{~g})$ and in females: $40 \mathrm{ml} @ 43 \%$ alcohol $=17.2 \mathrm{~g}$ alcohol per day $(43 \times 0.4=17.2 \mathrm{~g})$ (or $0.35 \mathrm{~g}$ alcohol $/ \mathrm{kg}$ body mass for males and females). Fasting blood specimens $(50 \mathrm{ml}$ per test day) were taken three times from the anterior cubital vein (1) after a 2-week washout period (baseline), (2) after the 28day red wine/brandy consumption period and (3) after the next 28-day wine/brandy consumption crossover period.

\subsection{Biochemical measurements and anti-oxidant status analysis}

Blood samples were drawn in the morning after a 12-hour overnight fast. Blood tests were performed $e x$ vivo, i.e. the wine or brandy was consumed by volunteers undergoing certain blood tests before and after each period of wine/brandy consumption to observe the effect that wine or brandy had on the specified biological systems (plasma levels of different lipoprotein fractions, oxidative and inflammatory status). A fasting lipid profile was determined using standard methods and biomarkers for oxidative stress and inflammation including a marker for lipid peroxidation, reduced to oxidized glutathione ratios (GSH:GSSG), oxygen radical absorbance capacity (ORAC), nitric oxide metabolic products and oxidized low density lipoproteins (Ox-LDL). Total phenolic acid content (TP) of plasma was determined using the Folin-Ciocalteau method with minor modification, to avoid plasma protein interference. Plasma antioxidant capacity was determined using the Trolox equivalent antioxidant capacity. Full lipid profiles and serum liver and kidney function indicators assessed to exclude possible toxicity induced by the consumption of the alcoholic beverages (red wine and brandy).

\subsection{Genetic studies}

DNA was extracted from whole blood using standard methods and amplified for further analysis using a real-time polymerase chain reaction (PCR)-based method. The genetic analysis was performed using the previously described CVD multi-gene assay [13] including 8 functional single nucleotide polymorphisms (SNPs) (Table 1) implicated in CVD and related disorders [10], with some modification to allow high-throughput analysis using TaqMan ${ }^{\circledR}$ technology. Direct DNA sequencing of PCR-amplified DNA was performed to verify the results obtained with the $\mathrm{ABI}^{\mathrm{TM}}$ TaqMan ${ }^{\circledR}$ SNP Genotyping assays using the Applied Biosystems $\left(\mathrm{ABI}^{\mathrm{TM}}\right)$ 7900HT Fast Real-Time PCR System.

\subsection{Statistical analysis}

Mixed model repeated measures ANOVA was used to analyse the crossover design for testing the effects of 
brandy and wine consumption relative to baseline measurements. Fisher least significant difference (LSD) was used for post hoc testing of differences between means. Analyses were done using Statistica 11.0 (StatSoft, USA). A $5 \%(p<0.05)$ significance level was used as a guideline for significant differences.

\section{Results and discussion}

The effects of alternative moderate consumption of red wine and brandy on biochemical and oxidative stress markers were compared with baseline measurements in an experimental population of 37 healthy adults that served as their own controls. The results of the blood biomarkers indicative of antioxidant activity and content, lipid peroxidation and redox status (GSH:GSSG) are shown in Table 2. When considering the plasma antioxidant capacity (ORAC) and the level of total polyphenols, no significant changes after the wine or brandy interventions were detected when compared to the baseline. This could be expected as the plasma used was from fasting samples and polyphenolic compounds have a relative short half-life (1-5 hrs) in plasma. No significant changes in plasma markers for lipid peroxidation (CDs, TBARS) when comparing the different interventions with the baseline were detected in this study. The level of total glutathione (tGSH) as an indicator of antioxidant capacity was significantly decreased only after the brandy intervention when compared to baseline $(p=0.002)$. This effect on the erythrocyte antioxidant capacity was not observed with the wine intervention and supports the protective effect of the antioxidants in red wine on the oxidative effect of alcohol on the body. Both brandy and wine consumption resulted in a significant increase in oxidised glutathione levels (more pronounced with brandy consumption) with a resultant decrease in glutathione redox status, but as mentioned with no associated increase in lipid peroxidation.

The most significant finding related to the response in the lipid profile was the effect of alcohol consumption on HDL-cholesterol (good cholesterol) levels. As shown in Fig. 1, both the wine and brandy intervention resulted in a significant increase in HDL-cholesterol levels, known to have a cardiovascular protective effect. This beneficial effect was observed in both the presence and absence of the APOE polymorphism, although to a lesser extent in participants with the e4 allele ( $p=0.027)$. The HDL-cholesterol raising effect

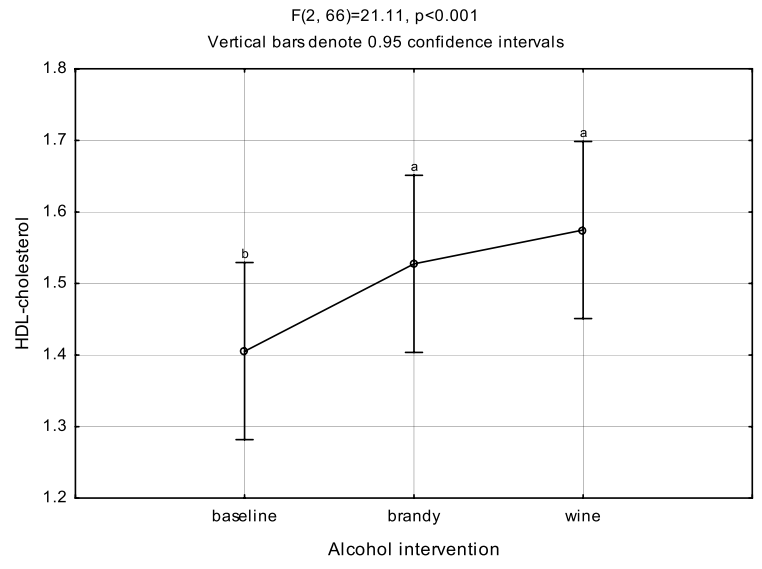

Fig. 1. Effect of alcohol consumption on HDL-cholesterol levels.

of alcohol was also modified by genetic variation in the methylenetetrahydrofolate reductase $(M T H F R)$ gene found to be associated with lower levels $(p=0.048)$. A similar association was previously reported in patients with familial hypercholesterolaemia [14] in relation to high homocysteine levels, known to reflect dysregulation of the methylation pathway.

An increase of total cholesterol and triglyceride levels was furthermore observed with alcohol intake in both the wine and the brandy intervention period. Since alcohol increases the uptake of iron from the diet, we investigated the potential influence of genetic variation in the haemochromatosis (HFE) gene on lipid levels in response to alcohol intake. Interestingly, a statistically significant increase was only observed in mutation-positive individuals for both cholesterol and triglyceride levels (Fig. 2a, b) This finding demonstrated an important gene-environment interaction and may explain why some individuals in the general population, but not all, experience an increase in cholesterol and triglyceride levels with alcohol intake. This negative finding with alcohol consumption may increase the risk in the minority of people with a genetic predisposition for iron overload and associated medical conditions.

Our results suggest that alcohol intake may be an important environmental trigger of the $H F E$ gene effect. The link between low-penetrance iron overload mutations and lipid abnormalities was also shown by Solanas-Barca et al. [15]. Most cases of primary hypertriglyceridemia are caused by interaction of unknown polygenes and environmental factors. Elevated iron storage is associated with the metabolic 
Table 2

Antioxidant status and oxidative stress parameters

\begin{tabular}{|c|c|c|c|c|c|c|c|}
\hline Group & $\begin{array}{c}\text { Conjugated } \\
\text { dienes }(\mu \mathrm{mol} / \mathrm{L})\end{array}$ & $\begin{array}{l}\text { TBARS } \\
(\mu \mathrm{mol} / \mathrm{L})\end{array}$ & $\begin{array}{c}\mathrm{tGSH} \\
(\mu \mathrm{mol} / \mathrm{L})\end{array}$ & $\begin{array}{c}{ }^{*} \mathrm{GSSG} \\
(\mu \mathrm{mol} / \mathrm{L})\end{array}$ & $\begin{array}{l}\text { GSH:GSSG } \\
\text { ratio }\end{array}$ & $\begin{array}{c}\text { ORAC } \\
(\mu \mathrm{mol} / \mathrm{L})\end{array}$ & $\begin{array}{c}\text { Total } \\
\text { polyphenols }(\mathrm{mg} / \mathrm{L})\end{array}$ \\
\hline Baseline & $128.1 \pm 18.9^{a}$ & $0.89 \pm 0.17^{\mathrm{a}}$ & $1006 \pm 177^{\mathrm{a}}$ & $18.8 \pm 30.6^{\mathrm{a}}$ & $145 \pm 108^{a}$ & $6193 \pm 684^{a}$ & $158 \pm 15^{\mathrm{a}}$ \\
\hline Wine & $133.5 \pm 19.0^{\mathrm{a}}$ & $0.87 \pm 0.14^{\mathrm{a}}$ & $995 \pm 157^{a}$ & $\begin{array}{c}20.1 \pm 14.1^{\mathrm{b}} \\
(p=0.019)\end{array}$ & $\begin{array}{c}84 \pm 63^{b} \\
(p<0.001)\end{array}$ & $6147 \pm 793^{a}$ & $155 \pm 15^{\mathrm{a}}$ \\
\hline Brandy & $130.2 \pm 20.7^{\mathrm{a}}$ & $0.88 \pm 0.17^{\mathrm{a}}$ & $\begin{array}{l}964 \pm 144^{b} \\
(p<0.001)\end{array}$ & $\begin{array}{c}27.4 \pm 23.1^{b} \\
(p<0.001)\end{array}$ & $\begin{array}{l}76 \pm 90^{\mathrm{b}} \\
(p<0.001)\end{array}$ & $5926 \pm 580^{\mathrm{a}}$ & $153 \pm 16^{\mathrm{a}}$ \\
\hline
\end{tabular}

*Analysis based on log transformed values. Values in columns are averages \pm SD. Values followed by the same letter indicates $p>0.05$ (nonsignificance), while when letters differ indicates $p<0.05$ (significance). Abbreviations: TBARS, thiobarbituric acid reactive substances; tGSH, total glutathione; GSSG, oxidized glutathione; GSH:GSSG, ratio of reduced to oxidised glutathione; ORAC, oxygen radical absorbance capacity.
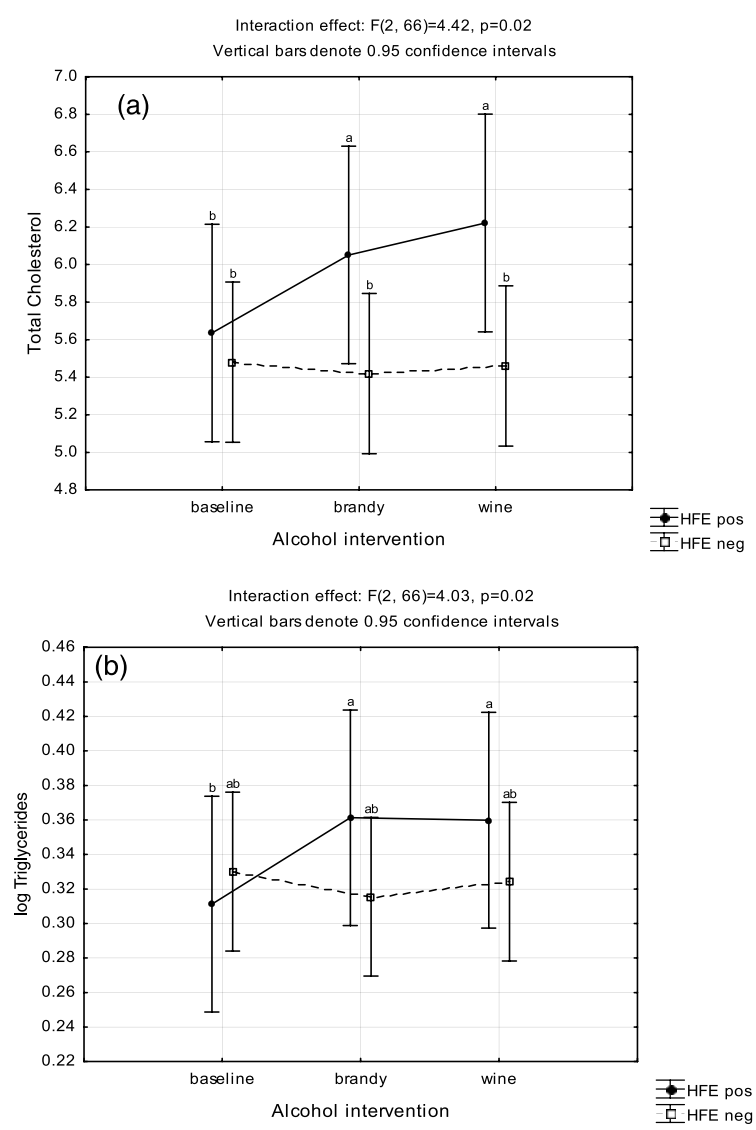

Fig. 2. a) Differential effect of alcohol consumption on total cholesterol levels in relation to genetic variation on the HFE (mutations C282Y and H63D) gene. b) Differential effect of alcohol consumption on triglyceride levels in relation to genetic variation on the $H F E$ (mutations C282Y and H63D) gene.

syndrome, diabetes and obesity, and all of these conditions are associated with hypertriglyceridemia. Variation in the $H F E$ gene, favouring iron overload and/or oxidative stress, could play an important part in the development of several phenotypes of primary hypertriglyceridemia. Fasting glucose concentrations did not change during the intervention periods, while there was a trend for the mean adjusted insulin values to decrease after both interventions. These findings support the protective effect of moderate alcohol intake on diabetes risk factors.

The genetic studies improved our understanding of the impact of hereditary factors on biochemical parameters of cardiovascular risk in relation to moderate alcohol consumption. Assessment of relatively common genetic variations may identify a subset of individuals in the general population who may not benefit from drinking alcohol as a consequence of gene-environment mismatches. Nutrition intervention may be more effective to lower cardiovascular risk when the genetic background is taken into consideration for identification of gene-environment interactions. This type of assessment may be particularly relevant to patients with features of the metabolic syndrome, which is characterised by three or more of the following features: central obesity, hypertension, glucose intolerance and dyslipidaemia (low HDLcholesterol and/or high triglyceride levels). Metabolic syndrome is associated with an increased risk of many chronic diseases including CVD and Alzheimer's disease. Realisation that the APOE e4 allele provides a genetic link between CVD and Alzheimer's disease highlighted the importance of addressing shared disease mechanisms before or early in disease development to optimize health in later life [10, 11]. Previous studies have shown that the APOE e4 allele increases the risk of Alzheimer's disease, especially when other CVD risk factors such as hypertension or obesity are also present. Alcohol furthermore increases the absorption of iron that is of specific concern in HFE gene mutation carriers with non-alcoholic fatty liver disease 
(NAFLD), the hepatic manifestation of the metabolic syndrome $[10,16]$.

\section{Conclusion}

Most large-scale epidemiological data on the cardiovascular benefits of moderate alcohol consumption come from North America, suggesting that there are no cardiovascular protective differences between drinking white or red wine or between alcohol taken as wine and other sources of alcohol [17]. This viewpoint is confirmed in a large and current meta-analysis [18]. The finding that alcohol consumption relates to an overall Jshaped mortality curve is no longer in doubt [19], thus establishing the benefits of moderate alcohol consumption. In the present study, only the brandy consumption adversely affected the level of total glutathione and not the red wine. Reduced glutathione is a powerful intracellular antioxidant that plays a vital role in stabilizing various enzymes.

The present study provided additional evidence that red wine, containing more non-alcoholic components than brandy, has the greatest health benefits. Moderate alcohol has a protective effect against cardiovascular disease risk factors, while red wine has an additional benefit that may be ascribed to the presence of polyphenol antioxidants in the wine. Although the antioxidant effects of red wine are well documented, it seems clear that alcohol intake may be contra-indicated in individuals with certain genetic alterations that occur relatively frequently in the general population [20]. Long-term intervention strategies for health promotion including lifestyle modification and safer daily drinking habits may therefore be more effective when guided from the genetic background. The healthful effects of wine may be enhanced by a healthy diet, such as the Mediterranean diet, suggesting that wine polyphenols have synergistic effects with compounds found in other types of food.

Early detection of genetic markers correlating with known biochemical pathological CVD risk factors would enable the development of individually tailored preventative medicine programmes. To address the limitations of genetics alone to fully account for the phenotypic expression of complex diseases, an integrative approach is required to effectively explore the relationship between known genetic markers of chronic disease and the associated lifestyle factors driving the disease process and clinical outcome.

\section{Acknowledgments}

Winetech and the Technology for Human Resources and Industry Program (THRIP) are thanked for financial support.

\section{References}

[1] Renaud S, de Lorgeril M. Wine, alcohol, platelets, and the French paradox for coronary heart disease. Lancet. 1992;339:1523-6.

[2] Trichopoulou A, Costacou T, Bamia C, Trichopoulos D. Adherence to a Mediterranean diet and survival in a Greek population. N Engl J Med. 2003;348:2599-608.

[3] Kant AK, Schatzkin A, Ziegler RG. Dietary diversity and subsequent cause-specific mortality in the NHANES I epidemiologic follow-up study. J Am Coll Nutr. 1995;14: 233-8.

[4] Empana JP, Tafflet M, Escolano S, Vergnaux AC, Bineau S, Ruidavets JB, Montaye M, Haas B, Czernichow S, Balkau B, Ducimetiere P. Predicting CHD risk in France: A pooled analysis of the D.E.S.I.R., three city, PRIME, and SU.VI.MAX studies. Eur J Cardiovasc Prev Rehabil. 2011;2:175-85.

[5] van Velden DP, Mansvelt EPG, Fourie E, Rossouw M, Marais AD. The cardioprotective effect of wine on human blood chemistry. Ann NY Acad Sci. 2002;957:337-40.

[6] Mansvelt EPG, Fourie E, Blackhurst D, Kotze T, Stofberg H, van der Merwe S, Kotze MJ, van Velden DP. The influence of a Mediterranean diet with and without red wine on the haemostatic and inflammatory parameters of subjects with the metabolic syndrome. S Afr J Enol Vitic. 2007;28:37-43.

[7] van Velden DP, van der Merwe S, Kidd M, Blackhurst D, Kotze MJ, Mansvelt EPG. The short-term influence of a Mediterranean-type diet and mild exercise with and without red wine on patients with the metabolic syndrome. S Afr J Enol Vitic. 2007;28:44-9.

[8] Kotze MJ, de Villiers WJS, Steyn K, Kriek JA, Marais AD, Langenhoven E, Herbert JS, Graadt van Roggen JF, van der Westhuyzen DR, Coetzee GA. Phenotypic variation among familial hypercholesterolemics heterozygous for either one of two Afrikaner founder LDL receptor mutations. Arterioscler Thromb. 1993;13:1460-8.

[9] Van Velden DP, Kotze MJ, Blackhurst D, Marnewick J, Kidd M. Health claims on the benefits of moderate alcohol consumption in relation to genetic profiles. J Wine Res. 2011;22:123-9.

[10] Kotze MJ, van Rensburg SJ. Pathology supported genetic testing and treatment of cardiovascular disease in middle age for prevention of Alzheimer's disease. Metab Brain Dis. 2012;27:255-66.

[11] Kotze MJ, van Velden DP, Botha K, Badenhorst CH, Avenant $\mathrm{H}$, van Rensburg SJ, Cronje FJ. Pathology-supported genetic testing directed at shared disease pathways for optimized health in later life. Personalized Med. 2013;10:497-507.

[12] Whitfield JB, Health AC, Madden PAF, Pergadia ML, Montgomery GW, Martin NG. Metabolic and biochemical effects of low-to-moderate alcohol consumption. Alcohol Clin Exp Res. 2013;37:575-86. 
[13] Kotze MJ, Thiart R. Genetics of dyslipidaemia. CME Journal. 2003;21:399-402.

[14] Real JT, Martinez-Hervas S, Garcia-Garcia AB, Chaves FJ, Civera M, Ascaso JF, Carmena R. Association of C677T polymorphism in MTHFR gene, high homocysteine and low HDL cholesterol plasma values in heterozygous familial hypercholesterolemia. J Atheroscler Thromb. 2009;16:815-20.

[15] Solanas-Barca M, Mateo-Gallego R, Calmarza P, Jarauta E, Bea AM, Cenarro A, Civeira F. Mutations in HFE causing hemochromatosis are associated with primary hypertriglyceridemia. J Clin Endocrin Metabolism. 2009;94:4391-7.

[16] Kotze MJ, Van Velden D, Van Rensburg SJ, Erasmus R. Pathogenic mechanisms underlying iron deficiency and iron overload: New insights for clinical application. J Int Fed Clin Chem Lab Med. 2009(02-01);1-15.
[17] Rimm EB, Klatsky A, Grobbee D, Stampfer MJ. Review of moderate alcohol consumption and reduced risk of coronary heart disease: Is the effect due to beer, wine, or spirits? BMJ. 1996;312:731-6.

[18] Ronksley PE, Brien SE, Turner BJ, Mukamal KJ, Ghali WA. Association of alcohol consumption with selected cardiovascular disease outcomes: A systematic review and meta-analysis. BMJ. 2011;342:d671.

[19] Pearson TA. Alcohol and heart disease. Circulation. 1996; 94:3023-5.

[20] Downer B, Zanjani F, Fardo DW. The relationship between midlife and late life alcohol consumption, $A P O E$ e4 and the decline in learning and memory among older adults. Alcohol Alcohol. 2013 Sep 18. [Epub ahead of print] 